\title{
The RAVE harvest: from the relation between abundances and kinematic of the Milky Way stars to tools for the abundance analysis of the spectra
}

\author{
Corrado Boeche ${ }^{1}$ and the RAVE collaboration \\ ${ }^{1}$ Astronomisches Rechen-Institut \\ Zentrum für Astronomie der Universität Heidelberg \\ Mönchhofstr. 12-14, 69120 Heidelberg, Germany \\ email: corrado@ari.uni-heidelberg.de
}

\begin{abstract}
RAVE is a spectroscopic survey of the Milky Way which collected more than 500,000 stellar spectra of nearby stars in the Galaxy. The RAVE consortium analysed these spectra to obtain radial velocities, stellar parameters and chemical abundances. These data, together with spatial and kinematic information like positions, proper motions, and distance estimations, make the RAVE database a rich source for galactic archaeology. I present recent investigations on the chemo-kinematic relations and chemical gradients in the Milky Way disk using RAVE data and compare our results with the Besançon models. I also present the code SPACE, an evolution of the RAVE chemical pipeline, which integrates the measurements of stellar parameters and chemical abundances in one single process.
\end{abstract}

Keywords. astronomical data bases: surveys, techniques: spectroscopic, Galaxy: disk, abundances

\section{The RAVE survey}

The RAdial Velocity Experiment (RAVE) is a large spectroscopic survey which observed stars of the Milky Way (Steinmetz et al. 2006) in the magnitude interval $9<I<12$ in the southern celestial hemisphere. After ten years of observations, the survey ended in April 2013, collecting 574,630 spectra of 483,330 stars. The spectra were obtained with the 1.2 meter UK Schmidt Telescope of the Australian Astronomical Observatory. The 150 optical fibres of the $6 \mathrm{dF}$ spectrograph allowed us to collect up to 130 spectra in one hour of exposure time. With a resolution of $\mathrm{R} \sim 7500$, the spectra centered on the near

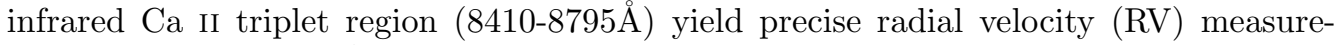
ments $\left(\sigma_{R V} \sim 2 \mathrm{~km} \mathrm{~s}^{-1}\right.$ at $\left.\mathrm{S} / \mathrm{N}>40\right)$. Beside, RAVE provides stellar parameters such as effective temperature, gravity, and metallicity (Zwitter et al. 2008, Siebert et al. 2011, Kordopatis et al. in preparation), and chemical abundances for the elements $\mathrm{Mg}, \mathrm{Al}, \mathrm{Si}$, Ti, Fe, and Ni (Boeche et al. 2011, Kordopatis et al. in preparation). Proper motions of the RAVE stars come from a variety of catalogues, such as Tycho2 (Høg et al., 2000), PPM-Extended catalogues PPMX and PPMXL (Röser et al., 2008, 2010) and the second and third U.S. Naval Observatory CCD Astrograph Catalog UCAC2 and UCAC3 (Zacharias et al., 2004). Distances have been estimated with different methods (Breddels et al. 2010, Zwitter et al. 2010, Burnett et al. 2011, Binney et al. in preparation). The kinematic information, combined with the distances and the chemical abundances, enables us to locate the RAVE stars in the six dimensional phase-space and the chemical space, making the RAVE database a rich mine for Galactic archaeology studies. 

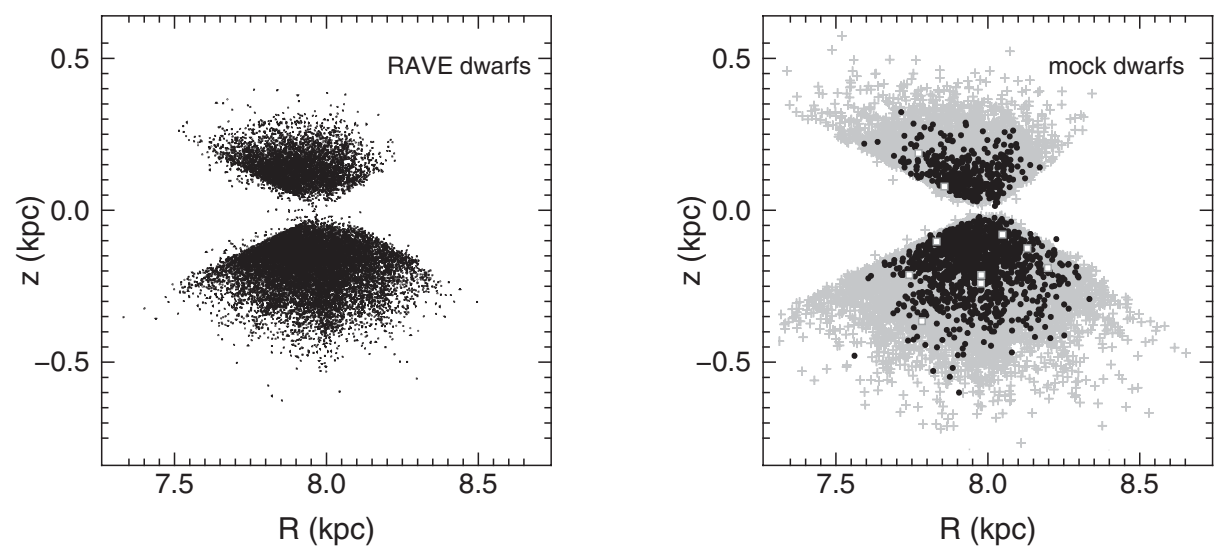

Figure 1. Spatial distribution of the RAVE sample (left) and the mock sample (right) on the meridional plane. For the mock sample the gray plus symbols, the black points and the gray open squares indicate the thin disc, the thick disc, and the halo stars, respectively.

RAVE is complementary to the Sloan Extension for Galactic Understanding and Exploration survey (SEGUE, Yanny et al. 2009). In fact, the two surveys observe in opposite hemispheres, at different magnitude ranges (SEGUE covers $14<g<20$ ), and at different resolution ( $\mathrm{R} \sim 2000$ for SEGUE). Because of the difference in magnitude, the SEGUE dwarf stars cover a volume similar to the one probed by the RAVE giant stars, making the comparison of the two samples crucial for the robustness of the results obtained with the two surveys.

\section{Radial chemical gradients of the Galaxy with RAVE: a comparison with the Besançon model}

We measured the chemical gradients of the Milky Way along its radius by using a sample of 19,962 RAVE dwarf stars (Boeche et al., submitted) selected to have spectra with $\mathrm{S} / \mathrm{N}>40$, effective temperature $5250<T_{\text {eff }}(K)<7000$, gravity $\log g>3.8$ dex and error in distance smaller than $30 \%$. The stars are also classified as normal stars by Matijevic et al. (2012) and have little or no continuum defects in their spectra. Since the RAVE dwarf stars cover a small Galacticentric distance range ( $\sim 0.6 \mathrm{kpc}$, see Fig. 1, left panel) a chemical gradient measured by using the actual positions of the stars will be necessarily poorly constrained. Thus, we extended the measurements to the Galactic radius range $\mathrm{R} \sim 4.5-9.5 \mathrm{kpc}$ by using the guiding radius $R_{g}$. To obtain $R_{g}$, the Galactic orbits of the stars have been integrated in the potential model n.2 by Dehnen \& Binney (1998). From the rotation curve and the integrated orbits we computed the guiding radius, $R_{g}$, and extract other orbital parameters such as apocentre, pericenter and maximum distance from the Galactic plane reached by the star $\left(Z_{\max }\right)$.

In order to avoid observational bias and compare the real Galaxy with models, we created a RAVE equivalent mock sample by using the stellar population synthesis code GALAXIA (Sharma et al., 2011), which uses analytical density profiles based on the Besançon model (Robin et al., 2003). The mock sample reproduces the RAVE selection function in $I$ magnitude and the target distribution on the sky. The same cuts in $\mathrm{S} / \mathrm{N}, \mathrm{T}_{\text {eff }}, \log g$ applied to the RAVE sample have been applied to the mock sample, which contains 26,198 entries (see Fig. 1, right panel). We measured the gradients of iron 


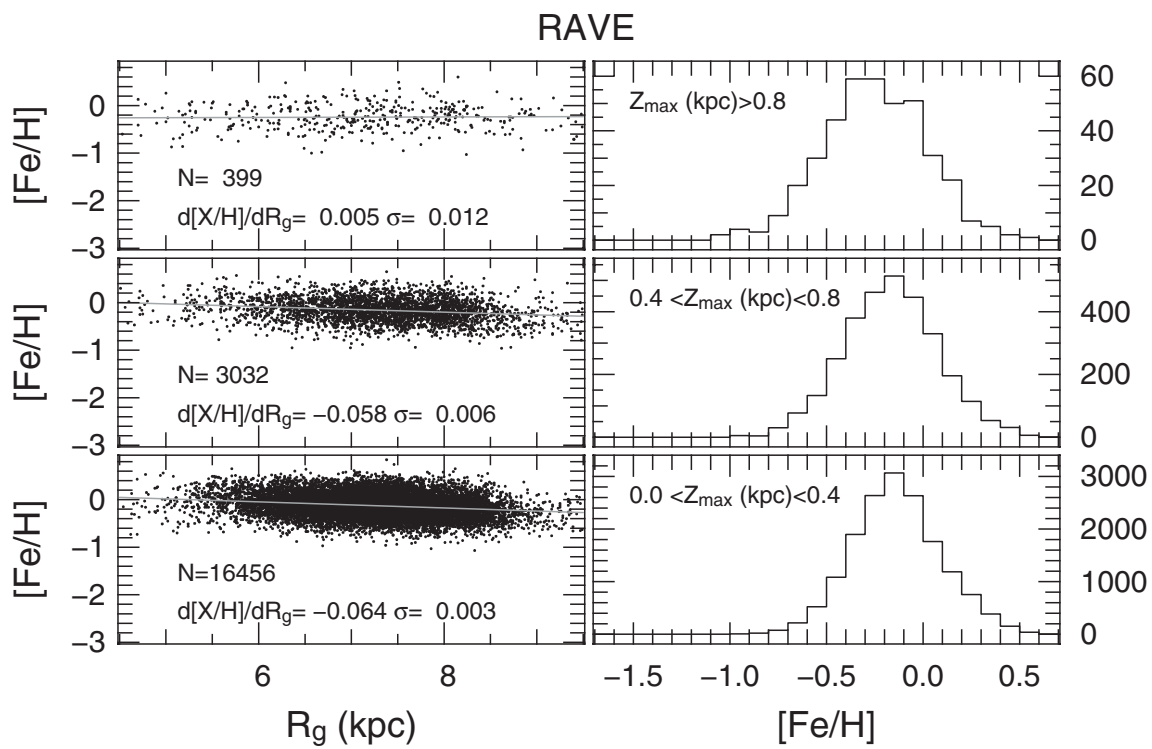

mock

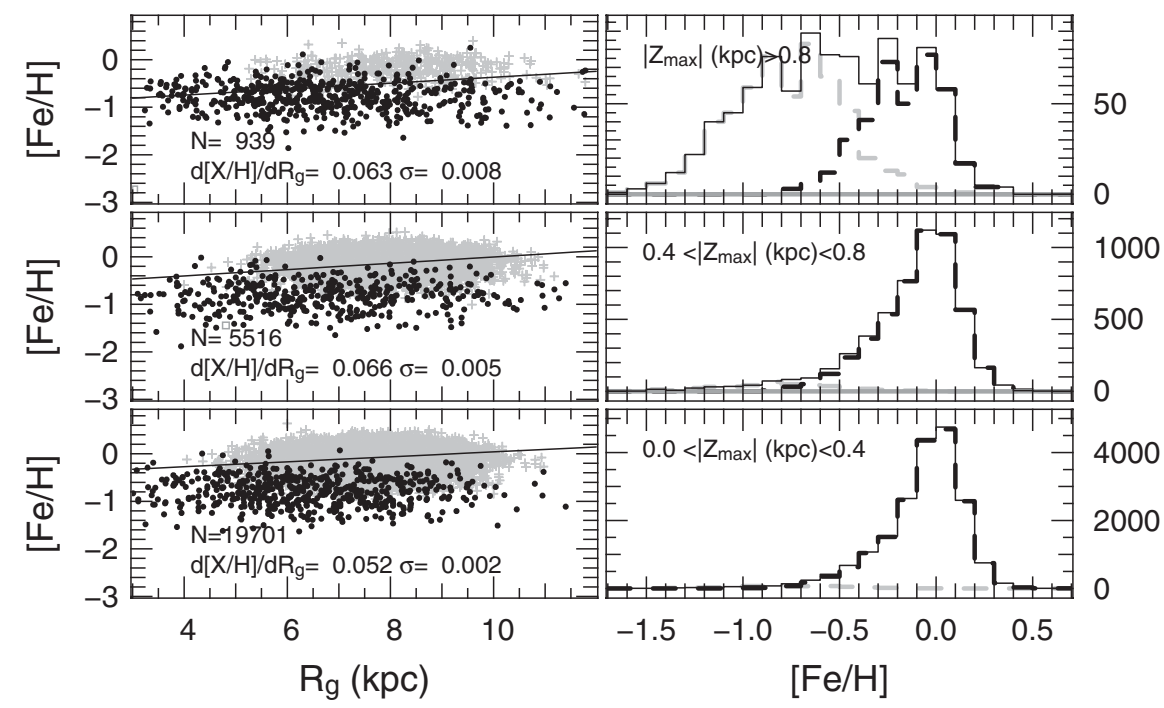

Figure 2. Distribution of the stars in the $\left(R_{g},[\mathrm{Fe} / \mathrm{H}]\right)$ plane (left panels) and $[\mathrm{Fe} / \mathrm{H}]$ distributions (right panels) of the three subsamples at different Zmax for the RAVE sample (top) and the mock sample (bottom). Symbols are as in Fig. 1. The $[\mathrm{Fe} / \mathrm{H}]$ distributions of the mock sample are traced with a dashed black line, a dashed gray line and a solid grey line for the thin disc, thick disc and halo stars, respectively. The overall distributions are represented by a black thin line.

abundance $[\mathrm{Fe} / \mathrm{H}]$ of these two samples at three different $\mathrm{Z}_{\max }$ values by dividing them in three subsamples: stars with $0.0<Z_{\max }(\mathrm{kpc}) \leqslant 0.4,0.4<Z_{\max }(\mathrm{kpc}) \leqslant 0.8$, and $Z_{\max }(\mathrm{kpc})>0.8$. The results are illustrated in Fig. 2 for the RAVE sample and the mock sample. 
The RAVE sample exhibits a negative gradient of $\mathrm{d}[\mathrm{Fe} / \mathrm{H}] / \mathrm{d} R_{g}=-0.064 \pm 0.002$ dex $\mathrm{kpc}^{-1}$ for $Z_{\max }<0.4 \mathrm{kpc}$, and becomes flat at $Z_{\max }>0.8 \mathrm{kpc}$. This result is in agreement with other previous works (Cheng et al. 2012, Pasquali \& Perinotto 1993). Conversely, the mock sample shows positive gradients at any $Z_{\max }$. These unrealistic gradients have several causes: i) the mock sample has an excess of thick disc stars (see the $[\mathrm{Fe} / \mathrm{H}]$ distributions in Fig. 2, bottom plot, right panels), ii) their mean metallicity appears too low with respect the RAVE sample, and iii) because of the larger asymmetric drift and the lower metallicity of the thick disc stars with respect to the thin disc stars, such stars are shifted toward lower $R_{g}$ and lower [Fe/H] (Fig. 2, bottom plot, left panels). The superposition of thin and thick disc stars mimics a positive gradient, the value of which depends on the ratio of thin/thick disc stars in the sample. Besides, the thin disc stars of the mock sample have the unrealistic gradient $\mathrm{d}[\mathrm{Fe} / \mathrm{H}] / \mathrm{d} R_{g}=0.00 \mathrm{dex} \mathrm{kpc}-1$ although the gradient in the actual Galactocentric distance, $R$, (assigned by the Besançon model) is $\mathrm{d}[\mathrm{Fe} / \mathrm{H}] / \mathrm{d} R=-0.07 \mathrm{dex} \mathrm{kpc}^{-1}$. This difference is due to the absence of a correlation between the kinematics and the metallicity. In fact, in the real Galaxy, stars with high eccentricity are more likely to be metal poorer, whereas in the Besançon model the metallicities of the stars are assigned considering their Galactocentric distances but regardless of their eccentricities (i.e. kinematics). The discrepancies between the RAVE sample and the mock sample can therefore be reduced by i) decreasing the density, ii) decreasing the vertical velocity, iii) increasing the metallicity of the thick disc in the Besançon model, and iv) assigning metallicities to the stars as a function of their kinematics, so that stars in high eccentricity orbits are on average metal poorer.

\section{SPACE: a new code for stellar parameters and chemical abundances estimations}

Large spectroscopic surveys face the challenge to process and analyse large databases of spectra in a reasonable time. This boosts the effort of developing new methods and automated tools for spectral analysis, which are also part of the survey's outcome. SPACE (which stands for Stellar PArameters and Chemical abundances Estimator) is one of RAVE's fruits, since it evolved from the RAVE chemical pipeline (Boeche et al. 2011). Both codes are based on 1D LTE (one dimensional, Local Thermodynamic Equilibrium) atmosphere models. The RAVE chemical pipeline derives chemical abundances from a normalised, radial velocity corrected spectrum, where stellar parameters such as $T_{\text {eff }}$, $\log g$ and a first guess of $[\mathrm{M} / \mathrm{H}]$ are provided by an external source. SPACE estimates stellar parameters and chemical abundances with no need of extra information but the normalized spectra and a first guess of the spectral resolution. SPACE does not rely on a library of synthetic spectra, nor does it measure equivalent widths (EWs) of isolated lines. Instead it relies on a list of lines with astrophisically corrected oscillator strengths, and on a library of Generalised Curves-Of-Growths (GCOGs) of such lines. The GCOG is a function in the 3-dimensional parameter space (PS, where the variables are $T_{\text {eff }}$, $\log g$, and abundance $[\mathrm{X} / \mathrm{H}]$ ), which describes the variation of the $\mathrm{EW}$ of a line in the PS. When $T_{\text {eff }}$ and $\log g$ are fixed, the GCOG reduces to the classical curve-of-growth. SPACE retrieves the EWs of the lines from the GCOGs of one point in the PS, reconstructs a spectrum model by assuming a Gaussian/Voigt line profile and varies the stellar parameters and chemical abundances, searching for the model that matches best the observed spectrum via $\chi^{2}$ minimization. To date, SPACE works in the stellar parameter ranges of $4000<T_{\text {eff }}(\mathrm{K})<7000,0.0<\log g($ dex $)<5.0$ and $\left.-2.5<\mathrm{Fe} / \mathrm{H}\right](\mathrm{dex})<0.5$. Extensions of the PS are possible. In principle, SPACE can work in any wavelength range when an appropriate line list and the corresponding library of GCOGs are provided. To 

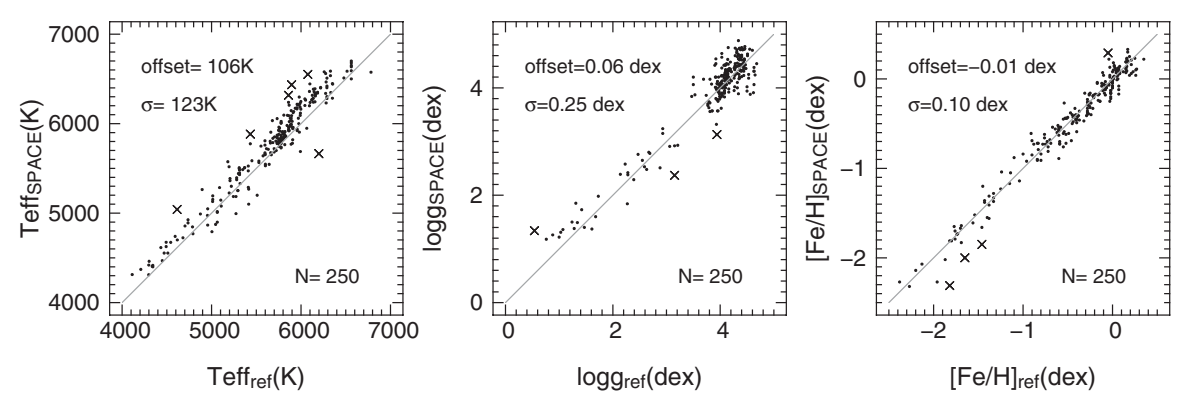

Figure 3. Stellar parameters derived by SPACE (y-axis) and the ELODIE reference parameter (x-axis) of ELODIE spectra reduced to spectral resolution $\mathrm{R}=5000, \mathrm{~S} / \mathrm{N} \sim 70$ and wavelength

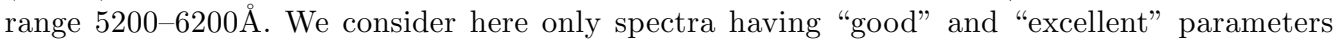
estimation flag in the ELODIE library.

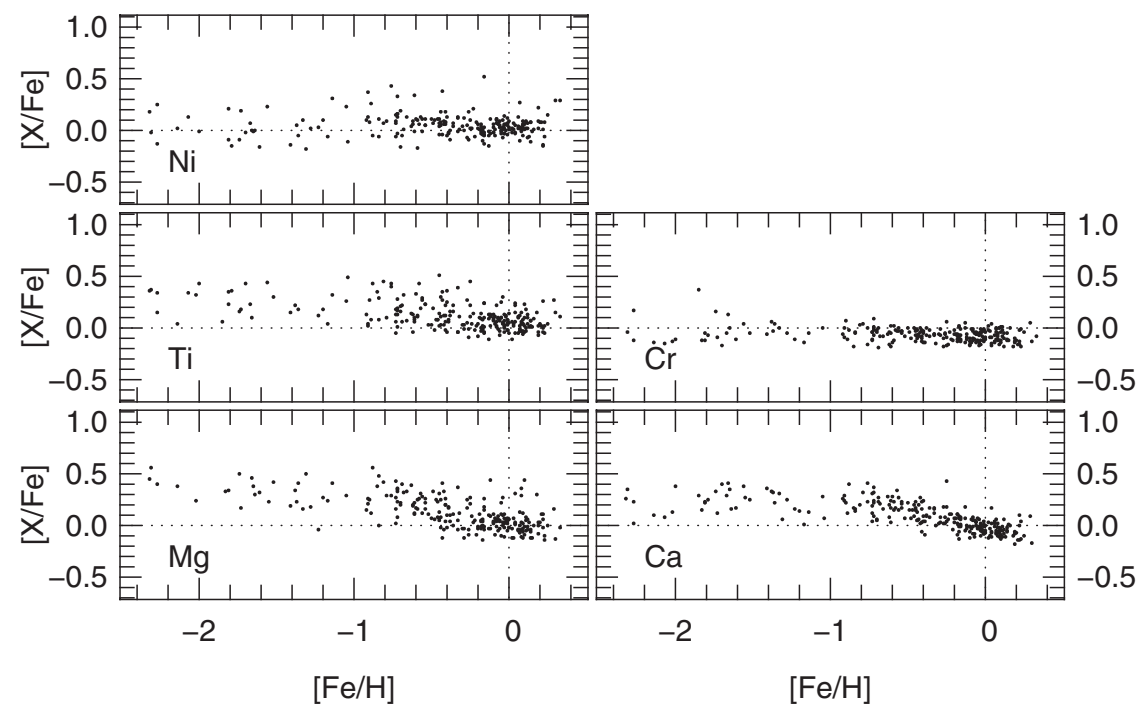

Figure 4. Chemical abundances of the ELODIE spectra reduced to spectral resolution $\mathrm{R}=$ $5000, \mathrm{~S} / \mathrm{N} \sim 70$ and wavelength range $5200-6200 \AA$ (the same used for Fig. 3).

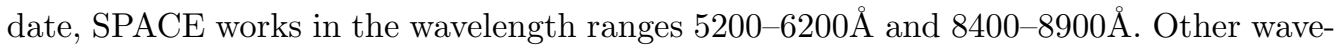
length ranges will be included soon. We tested SPACE by using spectra of the ELODIE spectral library (Prugniel et al., 2007) degraded to a resolution of $\mathrm{R}=5000$ and $\mathrm{R}=$

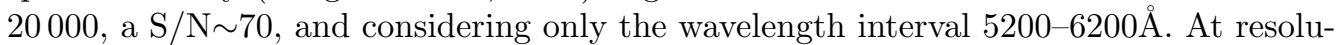
tion $\mathrm{R}=5000$ (Fig. 3) SPACE gives satisfactory results with $1 \sigma$ errors in $T_{\text {eff }}, \log g$ and $[\mathrm{Fe} / \mathrm{H}]$ of $\sim 120 \mathrm{~K}$ (with an offset of $+106 \mathrm{~K}$ ), 0.25 dex and 0.10 dex, respectively. Chemical abundances have uncertainties smaller than 0.1-0.2 dex (depending on the element) and correctly trace the enhancement of $\alpha$-elements with respect to iron (Fig. 4). At $\mathrm{R}=$ 20000 the errors do not seem smaller than the ones at $\mathrm{R}=5000\left(\sigma_{T_{e f f}}=172 \mathrm{~K}, \sigma_{\log g}=\right.$ $0.33 \mathrm{dex}$, and $\left.\sigma_{[\mathrm{Fe} / \mathrm{H}]}=0.10 \mathrm{dex}\right)$ because a not yet identified systematic error in $T_{\text {eff }}$ and $\log g$ affects the results. Nonetheless, the resulting chemical abundances look unexpectedly good, highlighting the gap in $\alpha$-enhancement between thin and thick disc stars in $[\mathrm{Ca} / \mathrm{Fe}]$ and $[\mathrm{Ti} / \mathrm{Fe}]$. More work and tests are needed in order to identify the causes of the systematic errors and to extend the working wavelength range. Once a stable version of the code exists, SPACE will be released to the scientific community as a tool for spectral analysis. 


\section{References}

Boeche, C., Siebert, A., Williams, M., et al., 2011, AJ, 142, 193

Breddels, M. A., Smith, M. C., Helmi, A., et al. 2010, A\& A, 511, A90

Burnett, B., Binney, J., Sharma, S., et al. 2011, AAP, 532, A113

Cheng, J. Y., Rockosi, C. M., Morrison, H. L., et al. 2012, ApJ, 746, 149

Høg, E., Fabricius, C., Makarov, V. V., et al. 2000, AAP, 355, L27

Dehnen, W., Binney, J. 1998, MNRAS, 294, 429

Matijevič, G., Zwitter, T., Bienaymé, O., et al. 2012, ApJS, 200, 14

Pasquali, A., Perinotto, M. 1993, A\&\&A, 280, 581

Prugniel, P., Soubiran, C., Koleva, M., \& Le Borgne, D. 2007, arXiv:astro-ph/0703658

Röser, S., Schilbach, E., Schwan, H., et al. 2008, A\&̛A, 488, 401

Röser, S., Demleitner, M., \& Schilbach, E. 2010, AJ, 139, 2440

Robin, A. C., Reylé, C., Derrière, S., \& Picaud, S. 2003, A\&A, 409, 523

Sharma, S., Bland-Hawthorn, J., Johnston, K. V., \& Binney, J. 2011, ApJ, 730, 3

Siebert, A., Williams, M. E. K., Siviero, A., et al. 2011, AJ, 141, 187

Steinmetz, M., Zwitter, T., Siebert, A., et al. 2006, AJ, 132, 16451

Yanny, B., Rockosi, C., Newberg, H. J., et al. 2009, AJ, 137, 4377

Zacharias, N., Urban, S. E., Zacharias, M. I., et al. 2004, AJ, 127, 3043

Zwitter, T., Siebert, A., Munari, U., et al. 2008, AJ, 136, 421

Zwitter, T., Matijevič, G., Breddels, M. A., et al. 2010, A\&SA, 522, 54

\section{Discussion}

JiNLIANG Hou: Can your SPACE software be used for spectra with different resolution?

Corrado Boeche: Yes, as shown in preliminary tests, SPACE gives reliable results between resolution $\mathrm{R}=5000$ and 20,000 . I will test the code at resolution $\mathrm{R}=2000$ to see if surveys like SEGUE and LAMOST can profit from it. Higher resolutions may be possible too and will be tested.

HANS-Günther LuDwiG: How do you handle the microturbulence when you calculate your library of curve-of-growths for SPACE?

Corrado Boeche: The microturbulence is determined by a formula (given in Boeche et al. 2011) which is function of $\mathrm{T}_{\mathrm{eff}}$ and $\log g$.

HANS-WALter RitTeR: How do your stellar atmospheric parameters compare to the official RAVE values?

Corrado Boeche: I did not test SPACE on RAVE spectra yet, but I will do it soon.

Giacomo Monari: In Fig. 2, top panels, what is the error in the slopes of the fit?

CorRado BoEche: The errors are reported in the text. 